\title{
Screening for differentially expressed genes between left- and right-sided colon carcinoma by microarray analysis
}

\author{
HONG ZHU ${ }^{1}$, TIAN-CONG WU ${ }^{1}$, WEI-QIONG CHEN ${ }^{1}$, LI-JUN ZHOU ${ }^{1}$, YUE WU ${ }^{1}$, \\ LIANG ZENG ${ }^{2}$ and HAI-PING PEI ${ }^{3}$

\begin{abstract}
${ }^{1}$ Department of Oncology, Xiangya Hospital, Central South University, Changsha, Hunan 410008; ${ }^{2}$ Department of Pathology, Hunan Tumor Hospital, Changsha, Hunan 410013; ${ }^{3}$ Department of Gastrointestinal Surgery, Xiangya Hospital, Central South University, Changsha, Hunan 410008, P.R. China
\end{abstract}

Received December 25, 2012; Accepted May 14, 2013

DOI: $10.3892 / \mathrm{ol} .2013 .1414$

\begin{abstract}
Left-sided colon carcinoma (LSCC) and right-sided colon carcinoma (RSCC) differ in their genetic susceptibilities to neoplastic transformation. The present study identified 11 genes that were differentially expressed in LSCC and RSCC by expression profiling with microarray analysis. Compared with RSCC, the human genes for L-lactate dehydrogenase B chain (LDHB), cyclin-dependent kinase 4 inhibitor D (CDKN2D), phosphatidylinositol-4-phosphate-3-kinase C2 domain-containing subunit $\alpha(\mathrm{PI} 3 \mathrm{KC} 2 \alpha)$, protocadherin fat 1 (FAT; a human protein that closely resembles the Drosophila tumor suppressor, fat) and dual specificity protein phosphatase 2 (DUSP2) were upregulated in LSCC. By contrast, genes for ubiquitin D (UBD), casein kinase-1 binding protein (CK1BP), synaptotagmin-13 (SYT1), zinc finger protein 560 (ZNF560), pleckstrin homology domain-containing family B member 2 (PLEKHB2) and IgGFc-binding protein (FCGBP) were downregulated in LSCC compared with RSCC. A quantitative polymerase chain reaction ( $\mathrm{qPCR}$ ) analysis revealed that the mRNA levels of $U B D$ and $C K 1 B P$ in LSCC were significantly lower compared with those in $\mathrm{RSCC}(\mathrm{P}=0.033$ and $\mathrm{P}=0.005$, respectively), whereas the mRNA levels of $\angle D H B$ and $C D K N 2 D$ in LSCC were significantly higher compared with those in $\operatorname{RSCC}(\mathrm{P}=0.008$ and $\mathrm{P}=0.017$, respectively). Western blot and immunohistochemical analyses demonstrated that the expression of CDKN2D in LSCC was significantly higher compared with that in RSCC, while the expression of UBD in LSCC was significantly lower compared with that in RSCC. The present study provides important insights into the understanding of the molecular genetic basis for the different biological behaviors observed between LSCC
\end{abstract}

Correspondence to: Professor Hai-Ping Pei, Department of Gastrointestinal Surgery, Xiangya Hospital, Central South University, 87 Xiangya Road, Changsha, Hunan 410008, P.R. China E-mail: peihaiping1966@163.com

Key words: left-sided colon carcinoma, right-sided colon carcinoma, cDNA microarray, differential gene expression and RSCC. These insights may therefore serve as a basis for the identification of novel colon cancer markers and therapeutic targets.

\section{Introduction}

Colon cancer is a significant cause of cancer-related morbidity and mortality, and is the third most fatal malignancy worldwide (1). In China and other economically developing countries, colon cancer incidence rates have increased over the past 20 years; most likely due to changes in the environment, individual lifestyle and nutritional habits (2). In certain high-prevalence regions, colon cancer has become the second leading cause of cancer-related mortality (3). It has been suggested that there are two distinct categories of colon cancer (CRC), i.e., CRC that is proximal or distal to the splenic flexure (4). A number of studies have demonstrated that right-sided (proximal) and left-sided (distal) colon tumors differ in their genetic susceptibilities to neoplastic transformation (5-9). Significant differences have been observed to exist between left-sided colon carcinoma (LSCC) and right-sided colon carcinoma (RSCC), with regard to epidemiological, biological and clinical data concerned with carcinogenesis and survival (10-13). Christodoulidis et al reported that the size of colonic tumors was significantly greater in RSCC compared with LSCC and that LSCC patients had a significantly improved overall 5-year survival rate compared with RSCC patients (10). Wray et al reported that LSCC presented at an earlier stage, had a lower tumor grade and independently decreased colorectal cancer-specific mortality compared with RSCC (11). Papagiorgis et al reported that RSCC had higher severity in terms of stage and grade compared with LSCC (13). However, the molecular genetic basis for the different biological behaviors between LSCC and RSCC remains unclear. Using cDNA microarray analysis, the present study explored the differentially expressed genes of LSCC and RSCC.

\section{Materials and methods}

Patients. From June 2007 to December 2008, 100 Han Chinese patients diagnosed with sporadic colon adenocarcinoma (LSCC, $\mathrm{n}=50$; RSCC, $\mathrm{n}=50$ ) were recruited from the Department of 
Table I. Baseline characteristics of patients.

\begin{tabular}{|c|c|c|c|}
\hline Characteristic & $\operatorname{LSCC}(n=50), n(\%)$ & $\operatorname{RSCC}(\mathrm{n}=50), \mathrm{n}(\%)$ & P-value \\
\hline \multicolumn{4}{|l|}{ Age (years) } \\
\hline$\leq 50$ & $15(30)$ & $21(42)$ & \multirow[t]{2}{*}{0.21} \\
\hline$>50$ & $35(70)$ & $29(58)$ & \\
\hline \multicolumn{4}{|l|}{ Gender } \\
\hline Male & $31(62)$ & $27(54)$ & \multirow[t]{2}{*}{0.54} \\
\hline Female & $19(38)$ & $23(46)$ & \\
\hline \multicolumn{4}{|c|}{ Tumor cell differentiation } \\
\hline High & $17(34)$ & $18(36)$ & \multirow[t]{3}{*}{0.57} \\
\hline Intermediate & $22(44)$ & $25(50)$ & \\
\hline Low & $11(22)$ & $7(14)$ & \\
\hline \multicolumn{4}{|c|}{ Lymph node metastasis } \\
\hline Yes & $26(52)$ & $21(42)$ & \multirow[t]{2}{*}{0.42} \\
\hline No & $24(48)$ & $29(58)$ & \\
\hline \multicolumn{4}{|l|}{ Liver metastasis } \\
\hline Yes & $11(22)$ & $7(14)$ & \multirow[t]{2}{*}{0.30} \\
\hline No & $39(78)$ & $43(86)$ & \\
\hline \multicolumn{4}{|l|}{ Tumor diameter (cm) } \\
\hline$\leq 5$ & $22(44)$ & $17(34)$ & \multirow[t]{2}{*}{0.31} \\
\hline$>5$ & $28(56)$ & $33(66)$ & \\
\hline \multicolumn{4}{|l|}{ Tumor invasion } \\
\hline Within muscle & $14(28)$ & $21(42)$ & \multirow[t]{2}{*}{0.14} \\
\hline Serosa and further & $36(72)$ & $29(58)$ & \\
\hline \multicolumn{4}{|l|}{ TNM stage } \\
\hline I and II & $11(22)$ & $16(32)$ & \multirow[t]{2}{*}{0.26} \\
\hline III and IV & $39(78)$ & $34(68)$ & \\
\hline
\end{tabular}

LSCC, left-sided colon carcinoma; RSCC, right-sided colon carcinoma.

General Surgery of Xiangya Hospital, Central South University (Changsha, China). All patients received complete resection of the tumor, without pre-operative chemotherapy or radiotherapy. The baseline characteristics of the patients are listed in Table I. The study was approved by the Ethical Committee of Xiangya Hospital, Central South University. Informed consent was obtained from all participants.

Reagents. The Nanjing University 22K Human Genome Array gene chip was purchased from CapitalBio Corp. (Beijing, China). The gene chip contained 21,522 70-mer oligonucleotide DNAs, each representing a human gene transcript. Among the 21,522 oligonucleotide DNAs, 21,329 were from the Human Genome Oligo Set, Version 2.1 (Eurofins MWG Operon, Huntsville, AL, USA) and the remaining 193 were synthesized by CapitalBio Corp. The anti-cyclin-dependent kinase 4 inhibitor D (CDKN2D) monoclonal (sc-71810) and goat anti-human ubiquitin D (UBD; sc-51082) antibodies were purchased from Santa Cruz Biotechnology, Inc. (Santa Cruz, CA, USA).

RNA isolation and microarray procedures. The total RNA was extracted from samples using TRIzol reagent (Invitrogen
Life Technologies, Carlsbad, CA, USA), and purified using the NucleoSpin RNA Clean-up kit (Macherey-Nagel GmbH and Co., KG, Düren, Germany). The total RNA was then transcribed into double stranded cDNA with a cDNA Synthesis kit obtained from Promega Corporation (Madison, WI, USA), and purified with a polymerase chain reaction (PCR) NucleoSpin Extract II kit (Promega Corporation). The double stranded cDNA was transcribed in vitro, compounded into cRNA, purified and then labeled with Cy5-dCTP (Amersham Pharmacia Biotech, Inc., Piscataway, NJ, USA) by the Klenow enzyme (Takara Bio, Inc., Otsu, Japan). Hybridization was performed at $42^{\circ} \mathrm{C}$ with the Nanjing University $22 \mathrm{~K}$ Human Genome Array gene chip (CapitalBio Corp.).

Chip scan and data analysis. The chip images were scanned using a LuxScan 10K-A double-channel laser scanner (CapitalBio Corp.). The signals referred to the unified data of the light intensity that were detected by the scanner and analyzed with the CapitalBio SpotData Pro 3.0 Microarray Image Analysis software (CapitalBio Corp.). The image signals were transmitted as digital signals, and then the data contained on the chips were normalized by the Lowess method (14). 
Quantitative (q)PCR. The total RNA were prepared using TRIzol reagent (Invitrogen Life Technologies) followed by purification with the Turbo DNA-free system (Ambion, Inc., Austin, TX, USA). The cDNA was synthesized using SuperScript II Reverse Transcriptase (Invitrogen Life Technologies). qPCR was performed using the LightCycler thermal cycler system (Roche Diagnostics GmbH, Mannheim, Germany) with the SYBR Green I kit (Roche Diagnostics $\mathrm{GmbH}$ ), according to the manufacturer's instructions. The results were normalized against those of the housekeeping gene, glyceraldehyde-3-phosphate dehydrogenase (GAPDH), in the same sample. The following primer sequences were used: $C D K N 2 D$ forward, 5'-CTCGCCGTCCTCCGGCTGAC-3' and reverse, 5'-AGCATGTCGACACTGGCGGC-3'; casein kinase-1 binding protein (C20orf35) forward, 5'-CССТTTCСТCСТCTTCATCC-3' and reverse, 5'-CССТTT CCTCCTCTTCATCC-3'; L-lactate dehydrogenase B chain $(L D H B)$ forward, 5'-TCCCGTGTCAACAATGGTAA-3' and reverse, 5'-CCCACAGGGTATCTGCACTT-3'; UBD forward, 5'-GGCACCTCCTCCAGGTGCGAA-3' and reverse, 5'-CAACACCCCATGCCCAGGGTG-3'; GAPDH forward, 5'-GTCAGTGGTGGACCTGACCT-3' and reverse, 5'-TGCTGTAGCCAAATTCGTTG-3'. Each sample was repeated in triplicate. The results are expressed as the mean \pm standard deviation.

Western blot analysis. Immunoblotting was performed with the respective antibodies. Briefly, extracted tumor tissues were homogenized and lysed in $0.1 \%$ Nonidet P-40 lysis buffer (0.1\% Nonidet P-40; 50 mM Tris-HCl, pH 7.4; 150 mM $\mathrm{NaCl}$; and $1 \mathrm{mM}$ EDTA). Equal quantities of protein for each sample were separated by $10 \%$ sodium dodecyl sulfate (SDS)-polyacrylamide gels and blotted onto a polyvinylidene difluoride microporous membrane (Millipore, Billerica, MA, USA). The membranes were incubated for $1 \mathrm{~h}$ with a 1:1,000 dilution of anti-CDKN2D monoclonal antibody (sc-71810) or goat anti-UBD antibody (sc-51082) (Santa Cruz Biotechnology, Inc.), and then washed and revealed using secondary antibodies with horseradish peroxidase conjugate $(1: 5,000 ; 1 \mathrm{~h})$. The peroxidase was revealed with an enhanced chemiluminescence (ECL) kit obtained from GE Healthcare Lifesciences (San Francisco, CA, USA). The proteins were quantified prior to being loaded onto the gel, and the equal loading of extracts was verified by an analysis of Ponceau coloration.

Immunohistochemistry. Paraffin-embedded tumor tissues were examined for CDKN2D or UBD expression. The immunostaining for CDKN2D and UBD was performed by utilizing the streptavidin-biotin-peroxidase method, according to the manufacturer's instructions (Beijing Zhongshan Golden Bridge Biotechnology Co., Ltd., Beijing, China). Briefly, 4- $\mu \mathrm{m}$ sections of paraffin-embedded specimens were de-paraffinized in xylene, hydrated in a degraded series of ethanol and heated in $0.01 \mathrm{M}$ citrate buffer for $10 \mathrm{~min}$ in a microwave oven. Subsequent to cooling for 20 min and washing in PBS, endogenous peroxidase was blocked with methanol containing $0.3 \%$ hydrogen peroxide for $30 \mathrm{~min}$, followed by incubation with phosphate buffered saline (PBS) for $30 \mathrm{~min}$. Next, the sections were incubated with the anti-CDKN2D or -UBD antibody at a dilution of 1:150, and stained using the avidin-biotin complex method. Coloration was developed by 3,3'-diaminobenzidine (DAB) containing $\mathrm{H}_{2} \mathrm{O}_{2}$, and the sections were counterstained with hematoxylin. Two pathologists, blinded to the clinical and pathological data, independently examined the slides by randomly selecting 10 high-power (x400) view fields in each sample and scoring the gene expression in the tumor cells, as previously described (15). Briefly, each tumor sample was administered a score according to the intensity of the nucleic or cytoplasmic staining ( 0 , no staining; 1 , weak staining; 2 , moderate staining; and 3, strong staining) and the extent of stained cells $(0 \%, 0 ; 1-10 \%, 1 ; 11-50 \%, 2 ; 51-80 \%, 3$; and $81-100 \%, 4)$. The extent of stained cells was classed as either negative, focally positive or diffusely positive, corresponding to the $0,1-80$ and $81-100 \%$ stained areas, respectively. The final immunoreactive score was determined by multiplying the staining intensity scores by the extent of staining scores, with a minimum score of 0 and a maximum score of 12 . Scores of 0-3 constituted negative staining, while scores of 4-12 indicated positive staining (15).

Statistical analysis. Statistical analyses were performed with SPSS for Windows 10.0 (SPSS, Inc., Chicago, IL, USA). Numerical data are presented as the mean \pm standard deviation. Comparisons were performed with the Student's t-test, following the assessment of normality and equality of variances. Categorical variables were compared using the $\chi^{2}$ test. $\mathrm{P}<0.05$ was used to indicate a statistically significant difference.

\section{Results}

As demonstrated in Table I, no significant differences were observed between the LSCC and RSCC patients in terms of the baseline characteristics, including age, gender, TNM staging, tumor cell differentiation, tumor size, tumor invasion and rate of lymph node or liver metastasis. The results indicated that the LSCC and RSCC groups were comparable at baseline.

Gene expression profiling of LSCC and RSCC was established using the Nanjing University 22K Human Genome Array gene chip (CapitalBio Corp.), which has been employed in previous studies for microarray analysis (16-17). The screening criteria for differentially expressed genes were as follows: score difference, $\geq 2$; LSCC/RSCC fold change, 0.5-2; and number of biological replicates, $\geq 3$. As demonstrated in Table II, 11 genes were identified to be differentially expressed between LSCC and RSCC. Compared with RSCC, genes for LDHB, CDKN2D, phosphatidylinositol-4-phosphate-3-kinase-C2 domain-containing subunit alpha (PI3KC2 $\alpha)$, protocadherin fat 1 (FAT) and dual specificity protein phosphatase 2 (DUSP2) were upregulated in LSCC. By contrast, genes for UBD, C20orf35, synaptotagmin-13 (SYT1), zinc finger protein 560 (ZNF560), pleckstrin homology domain-containing family B member 2 (PLEKHB2) and IgGFc-binding protein (FCGBP) were downregulated in LSCC, compared with RSCC.

As demonstrated in Fig. 1, qPCR revealed that the mRNA levels of $U B D$ and C20orf35 in LSCC were significantly lower 
Table II. Differentially expressed genes in LSCC and RSCC identified by microarray analysis.

A, Upregulated genes in LSCC and RSCC

\begin{tabular}{llcccl}
\hline GB accession & Name & Score & Fold change & Chromosome & GO biological process \\
\hline U40343 & CDKN2D & -2.456 & 0.347 & 19 & Cytoplasm \\
BG110199 & LDHB & 4.747 & 3.562 & 12 & Energy pathways \\
BX537504 & PI3KC2 $\alpha$ & 2.432 & 3.643 & 6 & Humoral defense mechanism \\
X87241 & FAT & 2.343 & 3.090 & 4 & Development \\
L11329 & DUSP2 & 2.076 & 2.567 & 2 & Macromolecule metabolism \\
\hline
\end{tabular}

B, Downregulated genes in LSCC and RSCC

\begin{tabular}{llcccc}
\hline GB accession & Name & Score & Fold change & Chromosome & GO biological process \\
\hline Y12653 & UBD & 2.785 & 4.926 & 6 & Organismal movement \\
AJ276469 & C20orf35 & -2.456 & 0.444 & 20 & Cell growth and/or maintenance \\
AB037848 & SYT13 & -2.322 & 0.200 & 11 & Coated vesicle \\
AK056548 & ZNF560 & -2.275 & 0.488 & 19 & Transcription, DNA-dependent \\
AK093730 & PLEKHB2 & -2.045 & 0.490 & 2 & - \\
D84239 & FCGBP & -2.026 & 0.187 & 19 & - \\
\hline
\end{tabular}

LSCC, left-sided colon carcioma; RSCC, right-sided colon carcinoma; GO, gene ontology; CDKN2D, cyclin-dependent kinase 4 inhibitor D; $L D H B$, L-lactate dehydrogenase B chain; $P I 3 K C 2 \alpha$, phosphatidylinositol-4-phosphate-3-kinase $\mathrm{C} 2$ domain-containing subunit $\alpha$; FAT, protocadherin fat 1; UBD, DUSP2, dual specificity protein phosphatase 2; ubiquitin D; C20orf35, casein kinase-1 binding protein; SYT13, synaptotagmin-13; ZNF560, zinc finger protein 560; $P L E K H B 2$, pleckstrin homology domain-containing family B member 2; FCGBP, IgGFc-binding protein.

A

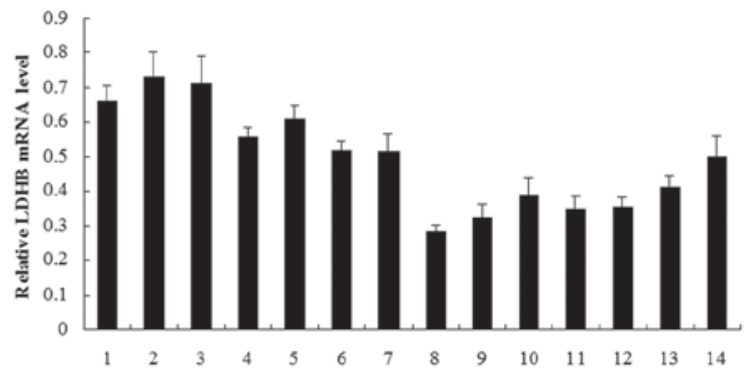

C

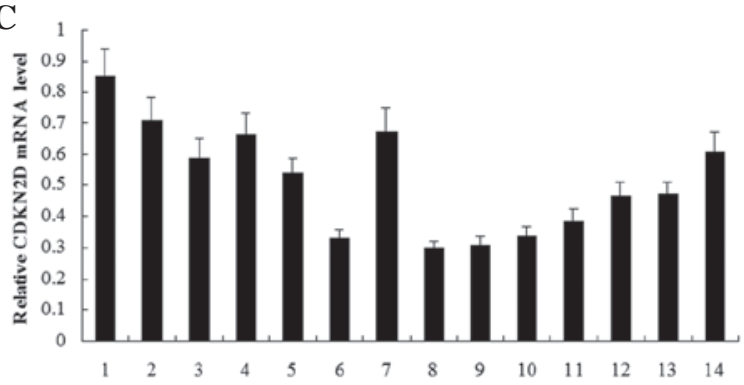

B

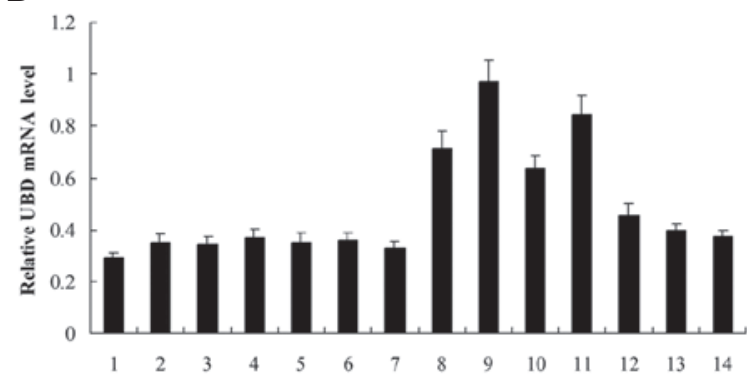

D

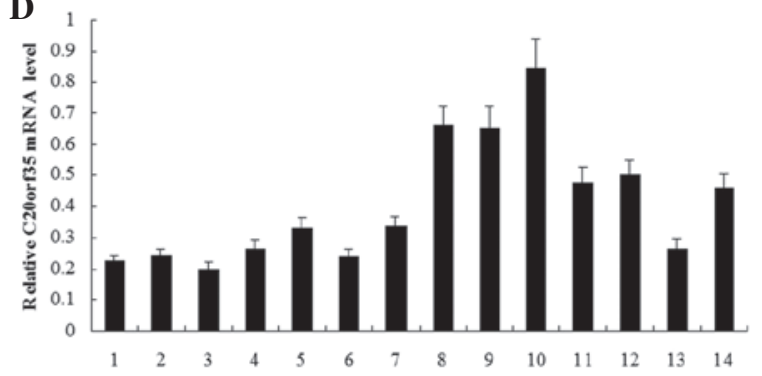

Figure 1. mRNA levels of differentially expressed genes in left-sided colon carcinoma (LSCC) and right-sided colon carcinoma (RSCC). Quantitative polymerase chain reaction (qPCR) was performed to examine the mRNA levels of differentially expressed genes in LSCC (sample nos. 1-7) and RSCC (sample nos. 8-14). (A) L-lactate dehydrogenase B chain $(L D H B)$; (B) ubiquitin $\mathrm{D}(U B D)$; (C) cyclin-dependent kinase 4 inhibitor $\mathrm{D}(C D K N 2 D)$; (D) casein kinase-1 binding protein (C20orf35). The mRNA level of each differentially expressed gene was normalized against that of the housekeeping gene, glyceraldehyde-3-phosphate dehydrogenase $(G A P D H)$, in the same sample. Each sample was repeated in triplicate and results are expressed as the mean \pm standard deviation.

than those in $\mathrm{RSCC}(\mathrm{P}=0.033$ and $\mathrm{P}=0.005$, respectively), while those of $\angle D H B$ and $C D K N 2 D$ were significantly higher in LSCC than those in RSCC $(\mathrm{P}=0.008$ and $\mathrm{P}=0.017$, respec- tively), thus confirming the results of the microarray analysis. The western blot analyses demonstrated that the protein level of CDKN2D in LSCC was significantly higher than that in RSCC 
Table III. Immunohistochemical detection of UBD and CDKN2D expression in LSCC and RSCC.

\begin{tabular}{|c|c|c|c|c|c|c|c|}
\hline & \multirow[b]{2}{*}{$\mathrm{n}$} & \multicolumn{2}{|c|}{ CDKN2D } & \multirow[b]{2}{*}{ P-value } & \multicolumn{2}{|c|}{ UBD } & \multirow[b]{2}{*}{ P-value } \\
\hline & & Positive, $\mathrm{n}$ & Positive rate $(\%)$ & & Positive, $n$ & Positive rate $(\%)$ & \\
\hline RSCC & 50 & 16 & 32 & 0.016 & 38 & 76 & 0.007 \\
\hline LSCC & 50 & 28 & 56 & & 25 & 50 & \\
\hline
\end{tabular}

UBD, ubiquitin D; CDKN2D, cyclin-dependent kinase 4 inhibitor D; LSCC, left-sided colon carcioma; RSCC, right-sided colon carcinoma.

A

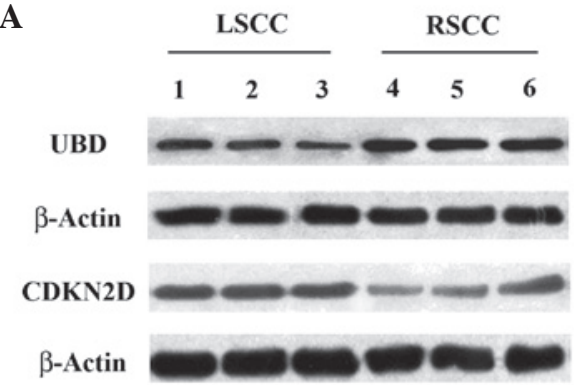

B

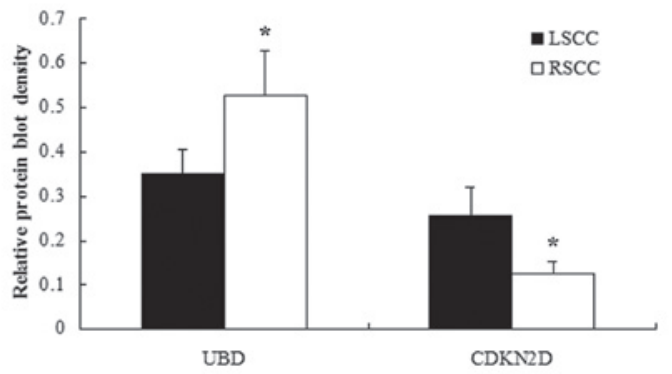

Figure 2. Western blot analysis of ubiquitin D (UBD) and cyclin-dependent kinase 4 inhibitor D (CDKN2D) expression in left-sided colon carcinoma (LSCC) and right-sided colon carcinoma (RSCC). (A) LSCC and RSCC tissue lysates were subject to western blot analysis for UBD and CDKN2D expression. $\beta$-actin blotting was used as a loading control. (B) UBD, CDKN2D and $\beta$-actin blots were measured by densitometry. The densities of the UBD and CDKN2D blots were normalized against that of $\beta$-actin, to obtain a relative UBD or CDKN2D blot density. ${ }^{*} \mathrm{P}<0.05$ compared with LSCC.

$(\mathrm{P}=0.041)$, while that of UBD in LSCC was significantly lower compared with that in RSCC $(\mathrm{P}=0.029)$ (Fig. 2). The immunohistochemical analyses revealed that the positive staining rate of CDKN2D in LSCC was significantly higher than that in RSCC ( $\mathrm{P}=0.016)$, while that of UBD in LSCC was significantly lower compared with that in RSCC (P=0.007; Table III; Fig. 3).

\section{Discussion}

Significant differences exist between LSCC and RSCC, with regard to epidemiological, biological and clinical data concerned with carcinogenesis and survival (10-13). A number of studies have suggested that LSCC and RSCC differ in their genetic susceptibilities to neoplastic transformation (5-9). Using expression profiling with microarray analysis, the present study identified 11 genes that were differentially expressed in LSCC and RSCC.

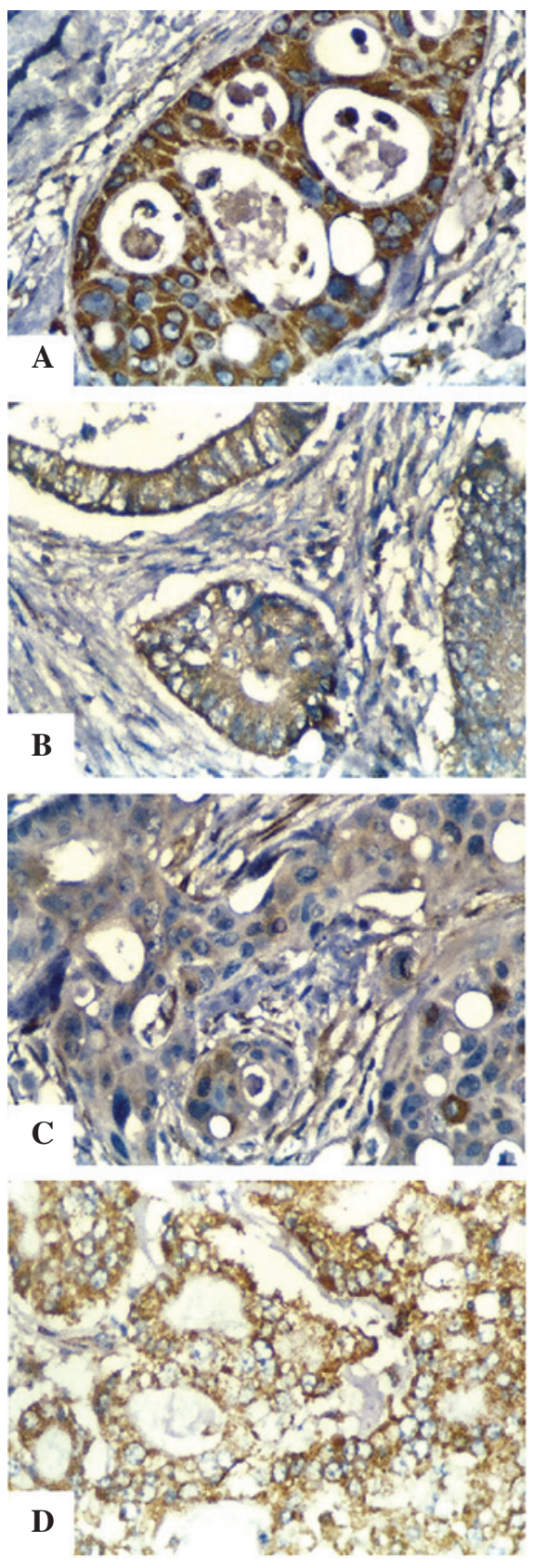

Figure 3. Immunohistochemical detection of ubiquitin D (UBD) and cyclin-dependent kinase 4 inhibitor D (CDKN2D) expression in left-sided colon carcinoma (LSCC) and right-sided colon carcinoma (RSCC). Immunohistochemical analyses were performed to determine (A and B) CDKN2D and (C and D) UBD expression in (A and C) LSCC and (B and D) RSCC. Sections were stained using the avidin-biotin complex method. The coloration was developed with 3,3'-diaminobenzidine (DAB), and the sections were then counterstained with hematoxylin. Positive staining for either CDKN2D or UBD appeared brown. Magnification, x400. 
Compared with RSCC, five genes were upregulated in LSCC; $L D H B, C D K N 2 D, P I 3 K C 2 \alpha, F A T$ and DUSP2. LDHB is an important enzyme in sugar metabolism. Griffini et al proposed that LDH may be involved in increasing anaerobic glycolysis in the metastatic foci of CRC in the liver, suggesting that the LDH activity may reflect the status of tumor metabolism (18). In the present study, LSCC demonstrated higher $L D H B$ expression than RSCC, implicating that the left and right side of the colon differ in their tumor metabolic activity, particularly with regard to anaerobic glucose metabolism. An RSCC is typically larger and has a poorer survival outcome compared with LSCC, which may be due to the fact that symptoms such as bleeding and pain occur later on in RSCC (10). CDKN2D has been demonstrated to induce tumor cell apoptosis through the cyclin D-CDK4/6-INK4-Rb pathway (19). The present study identified that LSCC had increased CDKN2D expression compared with RSCC, suggesting an additional mechanism for the differences in tumor size and survival outcome between LSCC and RSCC.

Compared with RSCC, six genes were downregulated in LSCC; UBD, C20orf35, SYT13, ZNF560, PLEKHB2 and $F C G B P$. Yan et al demonstrated that UBD may contribute to the progression of colon carcinogenesis and function as a novel prognostic indicator that may predict tumor recurrence in stage II and III patients following curative surgery (20). The results of the present study revealed that RSCC had higher $U B D$ expression than LSCC, suggesting that RSCC may have a poorer prognosis compared with LSCC, which is concordant with the results of a study by Christodoulidis et al (10). Studies have investigated the association between $F C G B P$ expression and the presence of tumors. O'Donovan et al revealed that while the FCGBP gene was constitutively expressed in normal thyroid tissue, its expression was significantly decreased in papillary and follicular thyroid carcinomas (21). The correlation between the expression of FCGBP, C2, FAT or DUSP2 and LSCC or RSCC remains unclear. Further studies are required to uncover the role of these genes in the pathogenesis and progression of LSCC and RSCC. Thus, the present results not only confirm those of previous studies, but also suggest potential targets for future studies on the pathogenesis and progression of colon cancer, which may serve as a basis for the identification of novel colon cancer markers and therapeutic targets. In addition, it may be useful to explore the potential interactions between the differentially expressed genes and well-studied oncogenes or tumor suppressor genes, such as V-Ki-ras2 Kirsten rat sarcoma viral oncogene homolog (KRAS), v-raf murine sarcoma viral oncogene homolog B1 (BRAF) and phosphatase and tensin homolog (PTEN).

In conclusion, using microarray analysis, the present study identified 11 genes that were differentially expressed between LSCC and RSCC. This therefore provided important insights into the understanding of the molecular genetic basis for the different biological behaviors that are evident between LSCC and RSCC.

\section{References}

1. Jemal A, Siegel R, Ward E, Hao Y, Xu J and Thun MJ: Cancer statistics 2009. CA Cancer J Clin 59: 225-249, 2009.
2. Zhang S, Cui Y, Weng Z, Gong X, Chen M and Zhong B: Changes on the disease pattern of primary colorectal cancers in Southern China: a retrospective study of 20 years. Int J Colorectal Dis 24: 943-949, 2009.

3. Jiang SX, Wang XS, Geng CH and Wang GY: Altering trend of clinical characteristics of colorectal cancer: a report of 3,607 cases. Ai Zheng 28: 54-56, 2009.

4. Suttie SA, Shaikh I, Mullen R, Amin AI, Daniel $T$ and Yalamarthi S: Outcome of right- and left-sided colonic and rectal cancer following surgical resection. Colorectal Dis 13: 884-889, 2011.

5. Bufill JA: Colorectal cancer: evidence for distinct genetic categories based on proximal or distal tumor location. Ann Intern Med 113: 779-788, 1990.

6. Elnatan J, Goh HS and Smith DR: C-KI-RAS activation and the biological behaviour of proximal and distal colonic adenocarcinomas. Eur J Cancer 32A: 491-497, 1996.

7. Bell SM, Scott N, Cross D, et al: Prognostic value of p53 overexpression and c-Ki-ras gene mutations in colorectal cancer. Gastroenterology 104: 57-64, 1993.

8. Breivik J, Meling GI, Spurkland A, Rognum TO and Gaudernack G: K-ras mutation in colorectal cancer: relations to patient age, sex and tumour location. Br J Cancer 69: 367-371, 1994.

9. Thibodeau SN, Bren G and Schaid D: Microsatellite instability in cancer of the proximal colon. Science 260: 816-819, 1993.

10. Christodoulidis G, Spyridakis M, Symeonidis D, Kapatou K, Manolakis A and Tepetes K. Clinicopathological differences between right- and left-sided colonic tumors and impact upon survival. Tech Coloproctol 14 (Suppl 1): S45-S47, 2010.

11. Wray CM, Ziogas A, Hinojosa MW, Le H, Stamos MJ and Zell JA: Tumor subsite location within the colon is prognostic for survival after colon cancer diagnosis. Dis Colon Rectum 52: 1359-1366, 2009

12. Benedix F, Kube R, Meyer F, Schmidt U, Gastinger I and Lippert H; Colon/Rectum Carcinomas (Primary Tumor) Study Group: Comparison of 17,641 patients with right- and left-sided colon cancer: differences in epidemiology, perioperative course, histology, and survival. Dis Colon Rectum 53: 57-64, 2010.

13. Papagiorgis P, Oikonomakis I, Karapanagiotou I, Wexner SD and Nikiteas N: The impact of tumor location on the histopathologic expression of colorectal cancer. J BUON 11: 317-321, 2006.

14. Yang YH, Dudoit S, Luu P, Lin DM, Peng V, Ngai J and Speed TP: Normalization for cDNA microarray data: a robust composite method addressing single and multiple slide systematic variation. Nucl Acids Res 30: e15, 2002.

15. Koo CL, Kok LF, Lee MY, et al: Scoring mechanisms of p16INK4a immunohistochemistry based on either independent nucleic stain or mixed cytoplasmic with nucleic expression can significantly signal to distinguish between endocervical and endometrial adenocarcinomas in a tissue microarray study. J Transl Med 7: 25, 2009.

16. Li Y, Basang Z, Ding H, et al: Latexin expression is downregulated in human gastric carcinomas and exhibits tumor suppressor potential. BMC Cancer 11: 121, 2011.

17. Roh SS, Lee MH, Hwang YL, et al: Stimulation of the extracellular matrix production in dermal fibroblasts by velvet antler extract. Ann Dermatol 22: 173-179, 2010.

18. Griffini P, Freitas I, Vigorelli E and Van Noorden CJ: Changes in the zonation of lactate activity in lobules of rat liver after experimentally induced colon carcinoma metastases. Anticancer Res 14: 2537-2540, 1994.

19. Sherr CJ and Roberts JM: CDK inhibitors: positive and negative regulators of G1-phase progression. Genes Dev 13: 1501-1512, 1999.

20. Yan DW, Li DW, Yang YX, et al: Ubiquitin D is correlated with colon cancer progression and predicts recurrence for stage II-III disease after curative surgery. Br J Cancer 103: 961-969, 2010.

21. O'Donovan N, Fischer A, Abdo EM, et al: Differential expression of IgG Fc binding protein (FcgammaBP) in human normal thyroid tissue, thyroid adenomas and thyroid carcinomas. J Endocrinol 174: 517-524, 2002. 